\title{
Identification of key genes in Gram-positive and Gram-negative sepsis using stochastic perturbation
}

\author{
ZHENLIANG LI ${ }^{1}$, YING ZHANG ${ }^{2}$, YALING LIU ${ }^{1}$, YANCHUN LIU ${ }^{1}$ and YOUYI LI ${ }^{1}$ \\ ${ }^{1}$ Intensive Care Unit, Pinggu Hospital Affiliated to Capital Medical University; ${ }^{2}$ Department of \\ Infection Diseases, Beijing Pinggu Hospital of Traditional Chinese Medicine, Beijing 101200, P.R. China
}

Received September 1, 2016; Accepted May 17, 2017

DOI: $10.3892 / \mathrm{mmr} .2017 .7013$

\begin{abstract}
Sepsis is an inflammatory response to pathogens (such as Gram-positive and Gram-negative bacteria), which has high morbidity and mortality in critically ill patients. The present study aimed to identify the key genes in Gram-positive and Gram-negative sepsis. GSE6535 was downloaded from Gene Expression Omnibus, containing 17 control samples, 18 Gram-positive samples and 25 Gram-negative samples. Subsequently, the limma package in $\mathrm{R}$ was used to screen the differentially expressed genes (DEGs). Hierarchical clustering was conducted for the specific DEGs in Gram-negative and Gram-negative samples using cluster software and the TreeView software. To analyze the correlation of samples at the gene level, a similarity network was constructed using Cytoscape software. Functional and pathway enrichment analyses were conducted for the DEGs using DAVID. Finally, stochastic perturbation was used to determine the significantly differential functions between Gram-positive and Gram-negative samples. A total of 340 and 485 DEGs were obtained in Gram-positive and Gram-negative samples, respectively. Hierarchical clustering revealed that there were significant differences between control and sepsis samples. In Gram-positive and Gram-negative samples, myeloid cell leukemia sequence 1 was associated with apoptosis and programmed cell death. Additionally, NADH:ubiquinone oxidoreductase subunit S4 was associated with mitochondrial respiratory chain complex I assembly. Stochastic perturbation analysis revealed that NADH:ubiquinone oxidoreductase subunit B2 (NDUFB2), NDUFB8 and ubiquinol-cytochrome
\end{abstract}

Correspondence to: Dr Zhenliang Li, Intensive Care Unit, Pinggu Hospital Affiliated to Capital Medical University, 59 Xinping North Road, Pinggu, Beijing 101200, P.R. China

E-mail: lizhenliang1973@hotmail.com

Abbreviations: DEGs, differentially expressed genes; FDR, false discovery rate; FC, fold-change; ICU, intensive care unit; PCC, Pearson's correlation coefficient; KEGG, Kyoto Encyclopedia of Genes and Genomes

Key words: Gram-negative sepsis, Gram-positive sepsis, similarity network, functional enrichment, stochastic perturbation c reductase hinge protein $(U Q C R H)$ were associated with cellular respiration in Gram-negative samples, whereas large tumor suppressor kinase 2 (LATS2) was associated with G1/S transition of the mitotic cell cycle in Gram-positive samples. $N D U F B 2, N D U F B 8$ and $U Q C R H$ may be biomarkers for Gram-negative sepsis, whereas LATS2 may be a biomarker for Gram-positive sepsis. These findings may promote the therapies of sepsis caused by Gram-positive and Gram-negative bacteria.

\section{Introduction}

Sepsis is a systemic and deleterious inflammatory response to noxious infection $(1,2)$. Sepsis causes $\sim 18$ million new cases and millions of deaths worldwide annually; therefore, it is a major cause of morbidity and mortality globally in critically ill patients $(3,4)$. The excessive activation of inflammation, complement and coagulation systems may damage the host's own tissues and organs, leading to multiple organ failure and death (5). In a group of patients diagnosed with sepsis, the most common causative agents are Gram-positive and Gram-negative bacteria $(6,7)$.

Tang et al (8) used the microarray expression profile of GSE6535 to identify the differentially expressed genes (DEGs) between patients with Gram-positive and Gram-negative sepsis with univariate $\mathrm{F}$ test according to the cut-off criteria of false discovery rate (FDR) $<0.05$ and Ilog fold-change (FC) $\mid>1.5$ and determined that Gram-positive sepsis and Gram-negative sepsis had a common host response at the transcriptome level in critically ill patients (8). However, a previous study illustrated the different mechanisms of sepsis caused by Gram-positive bacteria and Gram-negative bacteria. Hypoxia-inducible factor $1 \alpha$ and Kruppel-like factor 2 have been identified to be involved in Gram-positive endotoxin-mediated sepsis by regulating cellular motility and proinflammatory gene expression in myeloid cells (9). In Gram-negative bacteria-induced sepsis, it has been determined that the inhibition of caspase-1 and defective interleukin $1 \beta$ production are important immunological features (10). Additionally, $\alpha 2$-antiplasmin has been identified to be a protective mediator during Gram-negative sepsis by inhibiting bacterial growth, inflammation, tissue injury and coagulation (11). Furthermore, thrombomodulin-mediated protein $\mathrm{C}$ activation may contribute to protective immunity 
in severe Gram-negative sepsis by regulating inflammatory and procoagulant response (12). Despite the clinical importance of the disease and extensive research, no specific treatment is available for sepsis caused by Gram-positive and Gram-negative bacteria. Therefore, it is necessary to screen the biomarkers for sepsis.

The present study aimed to use the microarray data of Tang et al (8) to screen the DEGs in Gram-positive and Gram-negative samples compared with control samples using the limma package based on a wide range of thresholds $\left(\mathrm{P}<0.05\right.$ and $\left.\log _{2} \mathrm{FCl}>0.8\right)$. In addition, specific genes were collected as biomarkers for sepsis caused by Gram-positive and Gram-negative bacteria. A previous study has proposed that analyses based on differential statistical tests may lead to different outcomes (13). Therefore, the findings of the present study may differ to those of Tang et al (8).

\section{Materials and methods}

Microarray data. The microarray dataset of GSE6535 (8) was downloaded from the database of gene expression omnibus (www.ncbi.nlm.nih.gov/geo), which was sequenced on the platform GPL4274 NHICU Human 19K version 1.0. Probe annotation information for mapping the probes into gene symbols was also downloaded. From GSE6535 dataset, 17 neutrophil samples from patients without sepsis, 18 neutrophil samples from patients with Gram-positive sepsis, and 25 neutrophil samples from patients with Gram-negative sepsis were selected. Tang et al (8) obtained whole blood samples from critically ill patients on admission to the intensive care unit of Nepean Hospital (Sydney, Australia). Using Ficoll-Paque density gradient separation, neutrophils were isolated from the whole blood. The patients with sepsis were diagnosed retrospectively according to their medical record. According to the criteria established by Calandra and Cohen (14), the patients with sepsis were divided into Gram-positive and Gram-negative sepsis groups through assessing various clinical features, including physical examination and history and microbiological cultures, such as bronchoalveolar washings, urine, blood and cerebrospinal fluid. GSE6535 was deposited by Tang et al (8). The study of Tang et al was approved by the Ethics Committee of Nepean Hospital and written informed consent was provided by the patients or their families (8).

Data preprocessing and differential expression analysis. Based on the probe annotation information, probe IDs were converted into their corresponding gene symbols. The average value of multiple probes (that were corresponding to the same gene) was used as the gene expression value. To eliminate inherent expression differences between genes, the gene expression values were performed with Z-score normalization as previously described (15). Subsequently, the limma package version 3.32.2 in R (16) was used to screen the DEGs in the Gram-positive and Gram-negative samples compared with the control samples. The $\mathrm{P}<0.05$ and $\log _{2} \mathrm{FCl}$ $>0.8$ were used as the cut-off criteria for screening DEGs. Using the VennDiagram in R (17), the common DEGs between Gram-positive and Gram-negative samples, as well as the specific DEGs in Gram-positive samples or Gram-negative samples were identified. Gene Ontology (GO; www .geneontology.org) is a bioinformatics resource that may be used to classify gene product function using controlled, structured vocabularies (18). Using the Database for Annotation, Visualization and Integrated Discovery (DAVID) (19), GO functional enrichment analysis was performed on the common DEGs. The hierarchical cluster analysis of the specific DEGs in Gram-positive or Gram-negative samples was conducted using cluster version 3.0 software (20) and then visualized using TreeView tool version 3 (21).

Similarity network construction. Pearson's correlation coefficient (PCC) (22), which determines the correlation between two variables, was used to identify the positive or negative correlations among different samples, with the threshold of $\mid \mathrm{PCCl}>0.5$. Using Cytoscape version 2.8 software (23), a similarity network was constructed for the Gram-positive, Gram-negative and control samples.

Functional and pathway enrichment analyses. Kyoto Encyclopedia of Genes and Genomes (KEGG; www.genome.jp/kegg/), which integrates genomic, chemical and systemic functional information, is a useful resource for pathway mapping (24). Using the online tool DAVID (19), GO functional and KEGG pathway enrichment analyses were conducted for the DEGs. $\mathrm{P}<0.05$ was used as the threshold.

Identification of significantly differential functions using stochastic perturbations. The average expression value in Gram-positive or Gram-negative samples was calculated for each gene enriched in the same term (GO functions or KEGG pathways). Euclidean distance (25) was used to calculate the difference between the levels of all the terms between Gram-positive and Gram-negative samples, according to the following equation:

$$
\text { distance }=\sqrt{\sum_{i=1}^{T}\left(\bar{X}_{P i}-\bar{X}_{N i}\right)^{2}}
$$

Where distance represents the Euclidean distance between Gram-positive samples and Gram-negative samples; $\bar{X}_{p i}$ stands for the average expression value of gene $\mathrm{i}$ in Gram-positive samples; $\bar{X}_{p i}$ represents the average expression value of gene $\mathrm{i}$ in Gram-negative samples; and $\mathrm{T}$ indicates the gene number in each term.

Subsequently, stochastic perturbations were used (26) to determine the significance findings. The $18 \mathrm{Gram}$-positive and 25 Gram-negative samples were randomly sorted. Subsequently, 18 samples were randomly selected and defined as Gram-positive samples and the remaining 25 samples were defined as Gram-negative samples. The Euclidean distance between the newly defined Gram-positive samples and Gram-negative samples was recalculated. This was repeated for 10,000 times and the Euclidean distance for 10,000 perturbations were sorted from small to large and used as the background distribution. The ranking order of the initial Euclidean distance in the background distribution was calculated and converted to a $\mathrm{P}$-value. The terms with $\mathrm{P}<0.05$ were considered significantly differential functions between Gram-positive and Gram-negative samples. 
Table I. Top 10 upregulated and downregulated genes in patients with Gram-negative and Gram-positive sepsis.

\begin{tabular}{|c|c|c|}
\hline DEGs & $-\log _{2}(\mathrm{P}$-value $)$ & $\operatorname{logFC}$ \\
\hline \multicolumn{3}{|l|}{ Upregulated genes } \\
\hline$R P L 27$ & 3.735442 & 1.654937 \\
\hline TM4SF 1 & 2.691156 & 1.541493 \\
\hline SEC11A & 2.258718 & 1.518767 \\
\hline PLOD2 & 2.255042 & 1.494549 \\
\hline$U Q C R H$ & 2.154078 & 1.446315 \\
\hline$A F P$ & 1.889918 & 1.429409 \\
\hline$C D K 5 R A P 2$ & 3.965291 & 1.413672 \\
\hline EPB41LAA-AS1 & 4.122053 & 1.411759 \\
\hline SOD1 & 2.91784 & 1.40033 \\
\hline$C A N X$ & 4.013587 & 1.392226 \\
\hline \multicolumn{3}{|c|}{ Downregulated genes } \\
\hline$E V I 2 B$ & 4.312471 & -2.08489 \\
\hline$M M E$ & 5.521434 & -1.73605 \\
\hline$Z B P 1$ & 5.974694 & -1.56361 \\
\hline LITAF & 3.113137 & -1.54349 \\
\hline CYTH4 & 2.874971 & -1.53818 \\
\hline$F B X L 5$ & 2.808339 & -1.53 \\
\hline CHI3LI & 4.498941 & -1.45407 \\
\hline$Q P C T$ & 4.411504 & -1.45154 \\
\hline TREMI & 4.12983 & -1.43918 \\
\hline$M X D 1$ & 3.392031 & -1.41323 \\
\hline
\end{tabular}

B, Gram-positive

\begin{tabular}{llc}
\hline DEGs & $-\log _{2}(\mathrm{P}$-value $)$ & $\operatorname{logFC}$ \\
\hline Upregulated genes & & \\
SSBP1 & 1.896196279 & 1.5341469 \\
LAIR1 & 3.8569852 & 1.4491332 \\
MRPS18A & 2.377785977 & 1.4338711 \\
NDUFC2 & 3.935542011 & 1.4093212 \\
CTSC & 3.982966661 & 1.3851966 \\
MT1L & 2.991399828 & 1.3843636 \\
TM4SF1 & 2.249491605 & 1.3772427 \\
FCHSD2 & 1.694648631 & 1.301164 \\
CYP1B1 & 2.460923901 & 1.297874 \\
NDUFA4 & 1.876148359 & 1.267824 \\
Downregulated genes & & \\
CHI3L1 & 6.359519 & -1.92246 \\
EVI2B & 3.271646 & -1.71993 \\
MME & 4.36251 & -1.68036 \\
KCNB1 & 2.300162 & -1.56111 \\
LITAF & 2.415669 & -1.44533 \\
FUS & 2.767004 & -1.42285 \\
OPCT & 3.090444 & -1.38819 \\
CKAP4 & 4.251812 & -1.35146 \\
MCL1 & 4.221126 & -1.34034 \\
EFHC2 & 3.458421 & -1.32892 \\
\hline
\end{tabular}

DEGS, differentially expressed genes; FC, fold-change.
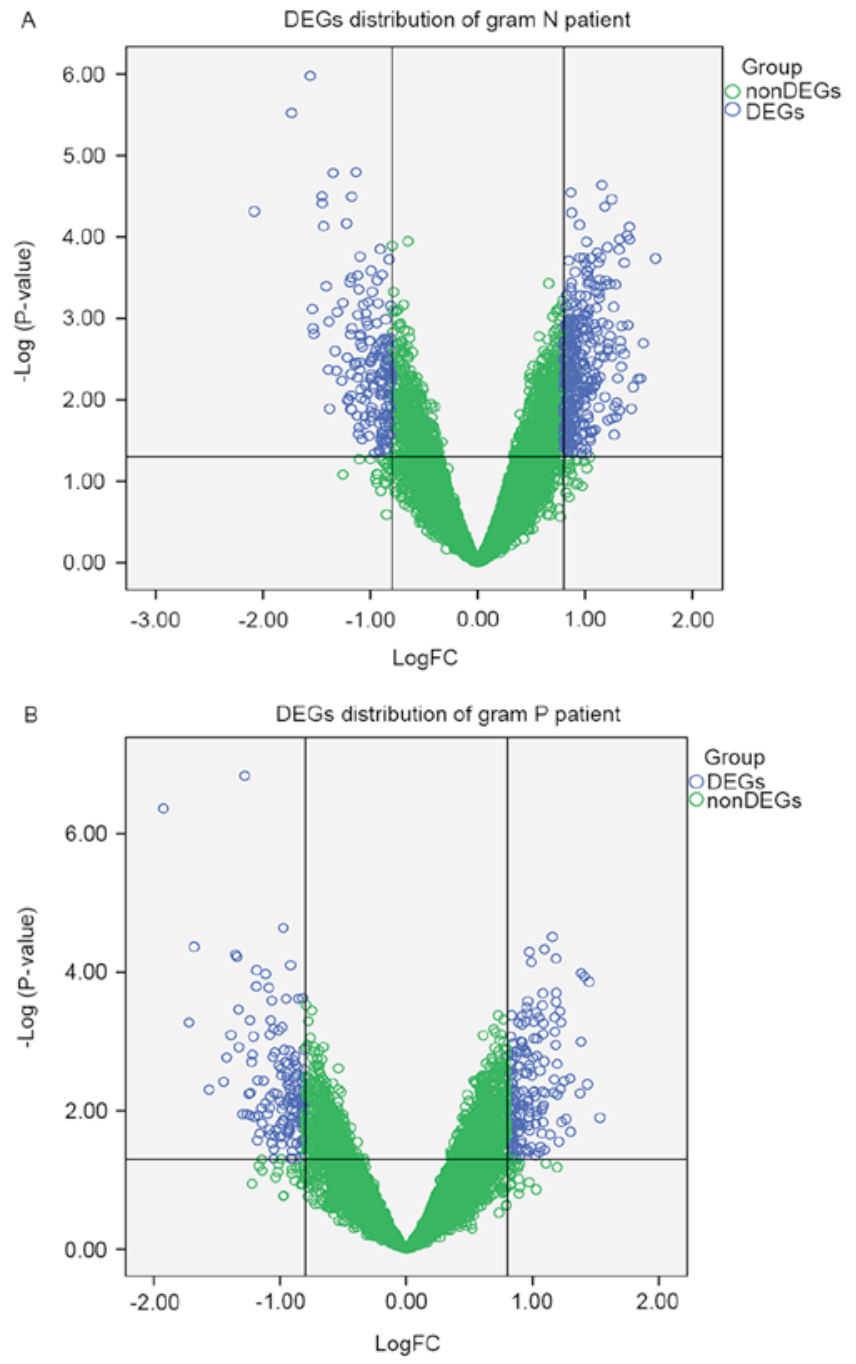

Figure 1. Volcano plots indicate the gene distribution of (A) Gram-negative samples and (B) Gram-positive samples. DEGs, differentially expressed genes; FC, fold-change.

\section{Results}

DEGs analysis. The gene distribution of Gram-negative (Fig. 1A) and Gram-positive samples (Fig. 1B) are presented using a volcano plot. Using the $\mathrm{P}<0.05$ and $\mid \log _{2} \mathrm{FCl}>0.8$ as thresholds, a total of 340 DEGs, including 181 upregulated genes, including large tumor suppressor kinase 2 (LATS2), NADH:ubiquinone oxidoreductase subunit S4 (NDUFS4) and 159 downregulated genes, including myeloid cell leukemia 1 (MCL1) and chitinase-like 1, were obtained in Gram-positive samples compared with control samples. A total of 485 DEGs were identified, 324 upregulated genes, including NDUFS4 and NADH:ubiquinone oxidoreductase subunit B2 (NDUFB2) and 161 downregulated genes, including MCL1 and ecotropic viral integration site $2 \mathrm{~B}$, were identified in Gram-negative samples compared with the control samples. The top 10 significantly upregulated genes and downregulated genes in the Gram-negative and Gram-positive samples are presented in Table I.

A total of 188 common DEGs, including 120 upregulated and 68 downregulated were identified between Gram-positive and Gram-negative samples. Additionally, 152 specific DEGs, 


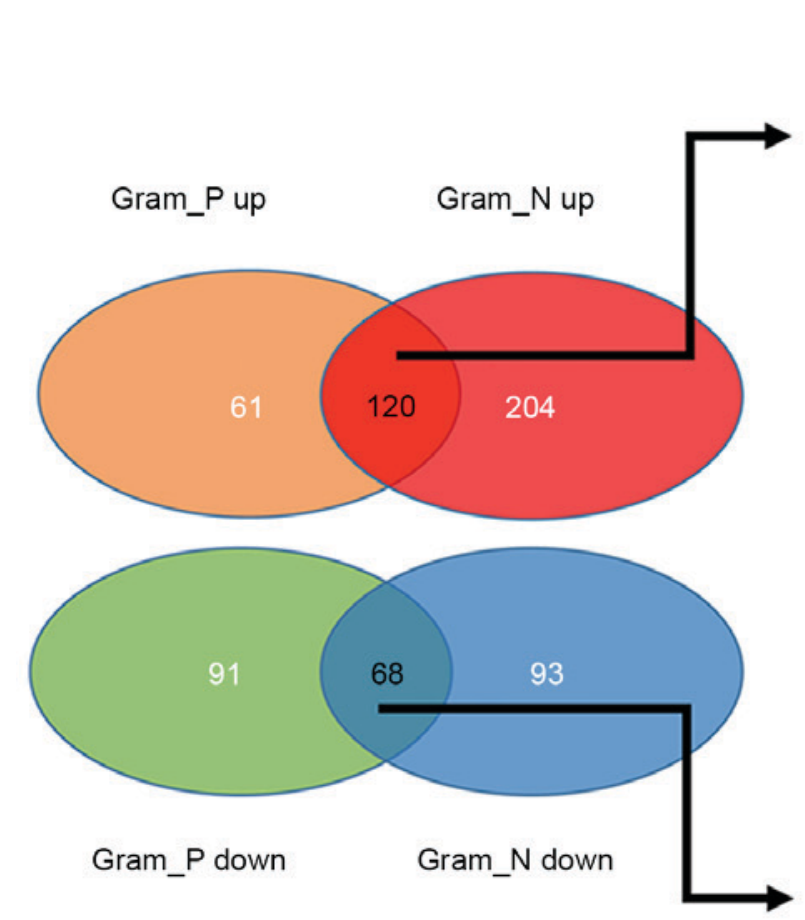

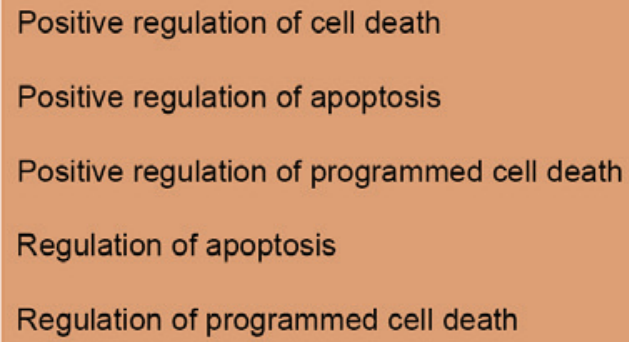

Figure 2. Summary of the common differentially expressed genes between Gram-negative and Gram-positive samples, and their respective enriched functions.

including 61 upregulated and 91 downregulated genes in the Gram-positive samples and 297 specific DEGs, including 204 upregulated and 93 downregulated genes in Gram-negative samples were also screened (Fig. 2). GO functional enrichment analysis was performed on the common DEGs, consisted of 120 upregulated and 68 downregulated genes in the Gram-positive and Gram-negative samples and the top 5 terms for each sample type were presented in Fig. 2. The findings revealed that the common upregulated genes were primarily associated with the regulation of apoptosis and cell death, whereas the common downregulated genes were primarily associated with cellular respiration (Fig. 2). Hierarchical cluster analysis of the specific DEGs revealed that there were significant differences between control and sepsis samples. However, no significant difference was identified between the Gram-positive and Gram-negative samples (Fig. 3).

Similarity network analysis. In the similarity network, positive associations were identified between the majority of the control and sepsis samples. However, negative associations were also identified between the control and sepsis samples (Fig. 4).

Functional and pathway enrichment analyses. Functional enrichment analysis was performed on the upregulated and downregulated genes in the Gram-positive or Gram-negative samples separately. For the downregulated genes in the Gram-positive samples and Gram-negative samples, MCL1 was significantly associated with the functions of apoptosis and programmed cell death regulation. For the upregulated genes in the Gram-positive and Gram-negative samples, NDUFS4 was significantly associated with mitochondrial respiratory chain complex I assembly. Additionally, NDUFB2, NDUFB 8 and ubiquinol-cytochrome $c$ reductase hinge protein
$(U Q C R H)$ were significantly enriched in the functions of cellular respiration, ATP synthesis coupled electron transport and respiratory electron transport chain in Gram-negative samples. LATS2 was significantly associated with the G1/S transition of the mitotic cell cycle in Gram-positive samples (Tables II and III). KEGG pathway enrichment analysis was also conducted for up and downregulated genes in Gram-positive and Gram-negative samples (Tables IV and V). NDUFS4 was significantly enriched in the pathway of oxidative phosphorylation.

Significantly differential functions screening. Based on the Euclidean distance of the biological functions, as well as the P-values of the 10,000 stochastic perturbations between Gram-positive samples and Gram-negative samples, a total of 10 significantly differential functions were obtained, including cellular respiration $\left(\mathrm{P}<1.00 \times 10^{-8}\right.$, Euclidean distance $=1.156277)$, ATP synthesis coupled electron transport $\left(\mathrm{P}<1.00 \times 10^{-8}\right.$, Euclidean distance $\left.=1.156277\right)$ and $\mathrm{G} 1 / \mathrm{S}$ transition of mitotic cell cycle $(\mathrm{P}=0.015$, Euclidean distance $=$ 0.554799; Table VI).

\section{Discussion}

In line with the results of Tang et al (8), the present study determined that there was no significant difference in the expression profile between Gram-positive and gram-negative samples from hierarchical clustering analysis. In the Gram-positive and Gram-negative samples, the GO functional enrichment analysis revealed that MCL1 was significantly associated with the regulation of apoptosis and programmed cell death. A previous study has determined that the apoptosis of T-cells may induce the breakdown of defense mechanisms resulting in sepsis (27). Additionally, 


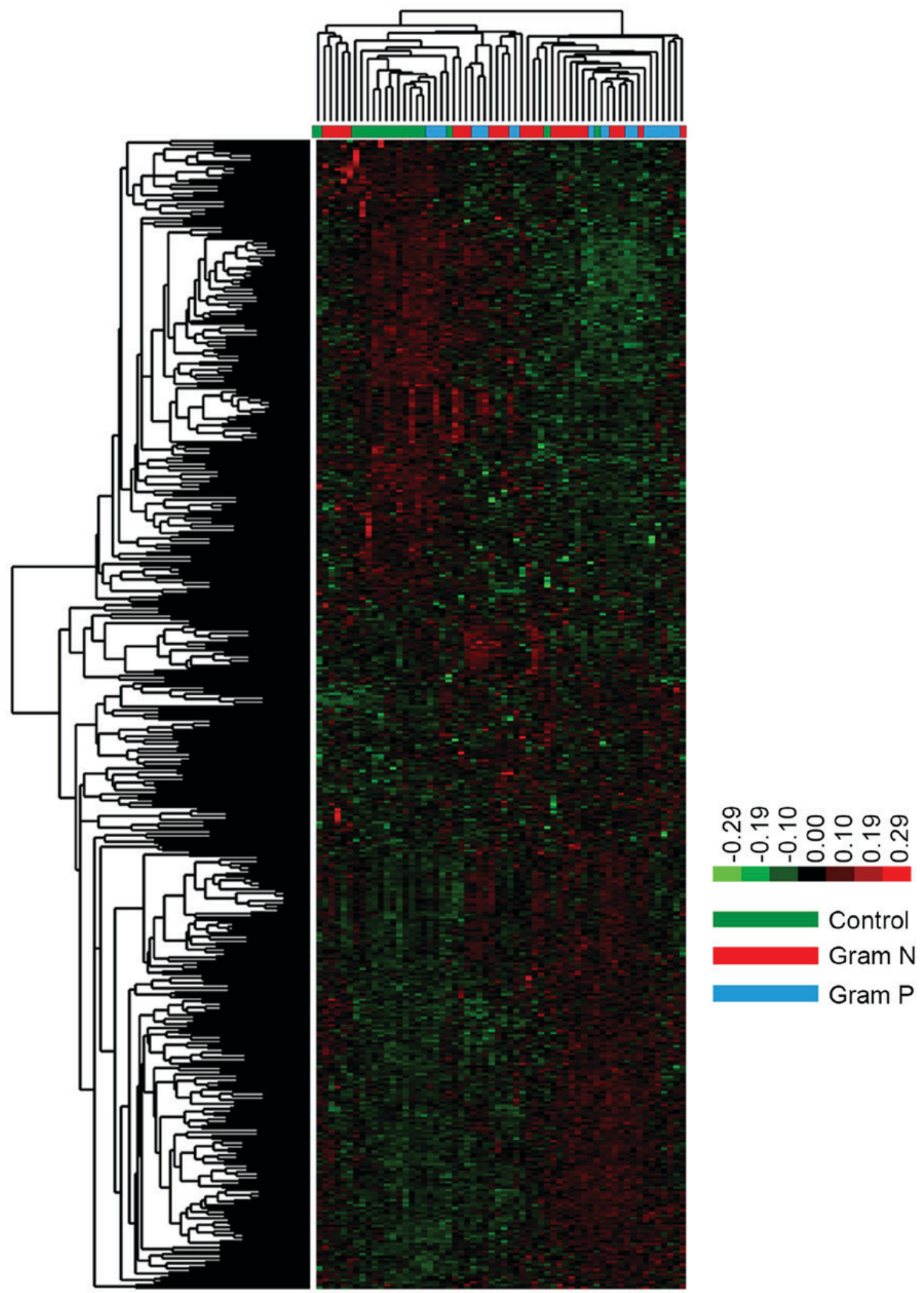

Figure 3. Hierarchical clustering of specific DEGs in Gram-P, Gram-N and control samples. Gram N, Gram-negative samples; Gram P, Gram-positive samples.

the inhibition of programmed cell death may reverse T-cell exhaustion and thus eradicate the invading pathogens which cause sepsis (28). Additionally, MCL1 may also be associated with the reduction of apoptosis of neutrophils in patients with sepsis (29). Therefore, it is possible for MCL1 to be involved in sepsis via the regulation of T-cell apoptosis and programmed T-cell death in both Gram-positive and Gram-negative sepsis.

Additionally, the present study also determined that NDUFS4 was significantly associated with mitochondrial respiratory chain complex I assembly. Mitochondrial dysfunction may lead to oxidative stress and failure of 


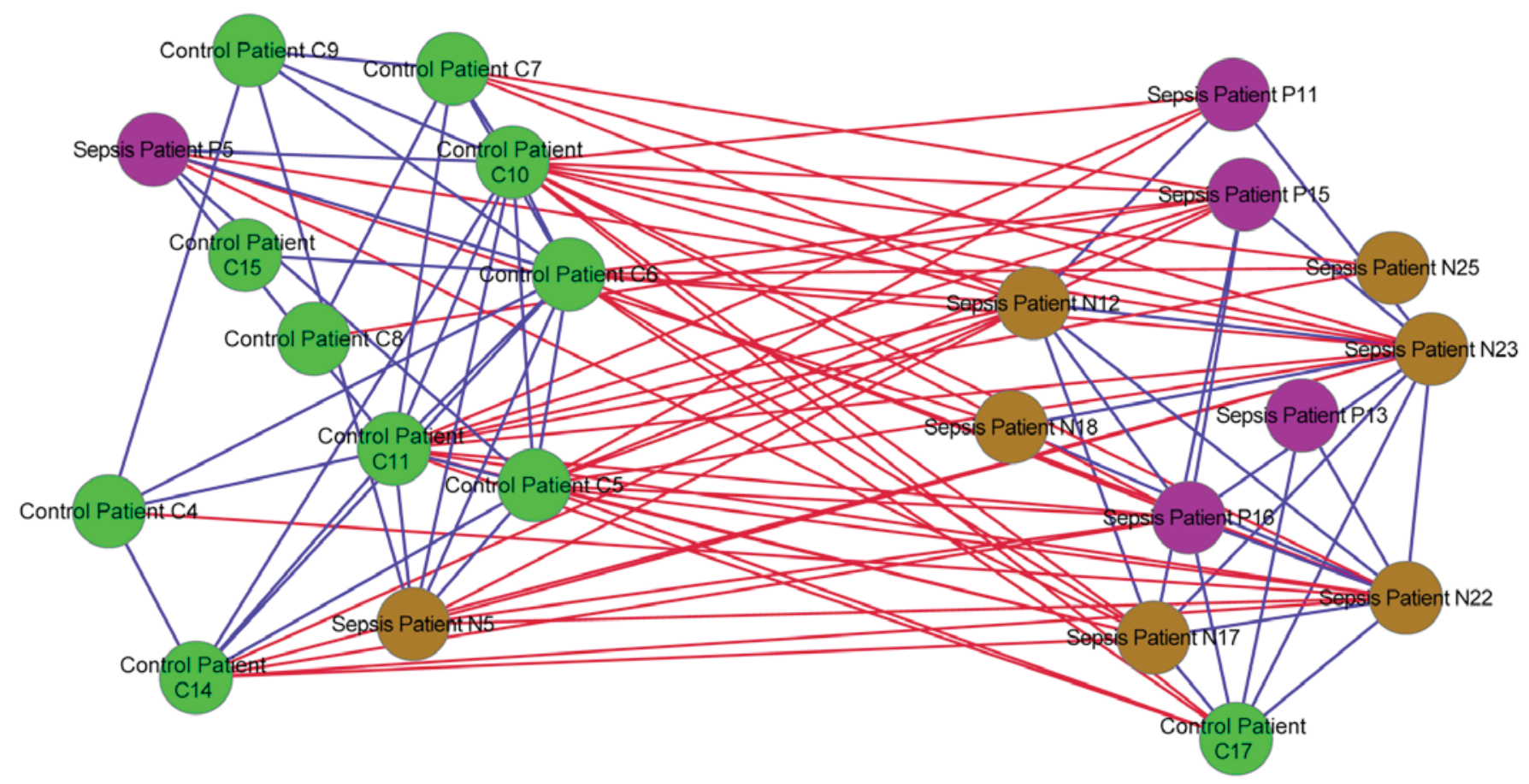

Figure 4. Correlation network of the Gram-positive, Gram-negative and control samples. Green circles indicate control samples, brown circles indicate Gram-negative samples and purple circles indicate Gram-positive samples. The red lines indicate a positive correlation between the two samples and blue lines indicate a negative correlation between the two samples.

energy production, which may result in organ dysfunction in sepsis (30). The KEGG pathway enrichment analysis revealed that NDUFS4 was significantly enriched in oxidative phosphorylation. Lee and Hüttemann (31) have determined that the inhibition of oxidative phosphorylation may lead to a reduction of the mitochondrial membrane potential, resulting in a lack of energy, which may cause organ failure and death in septic patients (31). NDUFS4 has been previously reported to be an important subunit of complex I which has a key role in oxidative phosphorylation (32). Additionally, NDUFS4 may participate in the regulation of sepsis induced by Gram-negative and Gram-positive bacteria through regulation of oxidative phosphorylation.

However, the present study identified specific DEGs in Gram-positive and Gram-negative samples compared with normal samples. According to the Euclidean distance and the stochastic perturbations performed between Gram-positive and Gram-negative samples, NDUFB2, NDUFB 8 and $U Q C R H$ were significantly upregulated in the Gram-negative samples, whereas they were not upregulated in the Gram-positive samples. In addition, functional annotation revealed that they were significantly associated with cellular respiration, ATP synthesis coupled electron transport and mitochondrial electron transport, ubiquinol to cytochrome $c$. NDUFB2 and NDUFB 8 are parts of the multisubunit mitochondrial NADH ubiquinone oxidoreductase (complex I) which has an important role in mitochondrial functioning $(33,34)$. A previous study determined that a dysfunction of respiratory chain complex I may be associated with reactive oxygen species (ROS) production (35). Additionally, previous studies reported that ROS are toxic oxygen-containing molecules that may damage the cells and the antioxidant defense system, which is the pathogenesis of sepsis $(36,37)$. UQCRH, which encodes the cytochrome b-c1 complex subunit 6 of complexes III (cytochrome $c$-oxidoreductase), is involved in the mitochondrial oxidative phosphorylation and the dysfunction of UQCRH may lead to breast and ovarian cancer by altering the function of the mitochondria $(38,39)$. To the best of our knowledge, this is the first study investigating the functions of NDUFB2, NDUFB 8 and $U Q C R H$ in Gram-negative bacteria-induced sepsis. The present study concluded that NDUFB2, NDUFB 8 and $U Q C R H$ may be involved in the Gram-negative bacteria-induced sepsis by altering mitochondrial oxidative phosphorylation and may also be potential targets for the treatment of Gram-negative bacterial sepsis.

In addition, the function of the G1/S transition of the mitotic cell cycle was also determined to be significantly different between the Gram-positive and Gram-negative samples. LATS2 was enriched in this function and was significantly upregulated in patients with Gram-positive sepsis, whereas it was not significantly expressed in Gram-negative patients. LATS2, encoding serine/threonine-protein kinase, has been identified to inhibit the G1/S transition in the cell cycle of tumor cells (40). Additionally, G1 cell cycle arrest may be important for the initiation of kidney injury in sepsis (41). Therefore, LATS2 may be associated with Gram-negative bacterial sepsis by the modulation of G1/S transition in cell cycle.

In conclusion, MCL1, NDUFS5 and NDUFS4 may be potential target genes for the treatment of Gram-positive and Gram-negative bacterial sepsis. Additionally, $N D U F B 2$, $N D U F B 8$ and $U Q C R H$ may also be associated with Gram-negative bacterial sepsis. LATS2 may contribute to the progression of Gram-negative bacterial sepsis. However, further studies are still required in order to elucidate their action mechanisms in sepsis. 


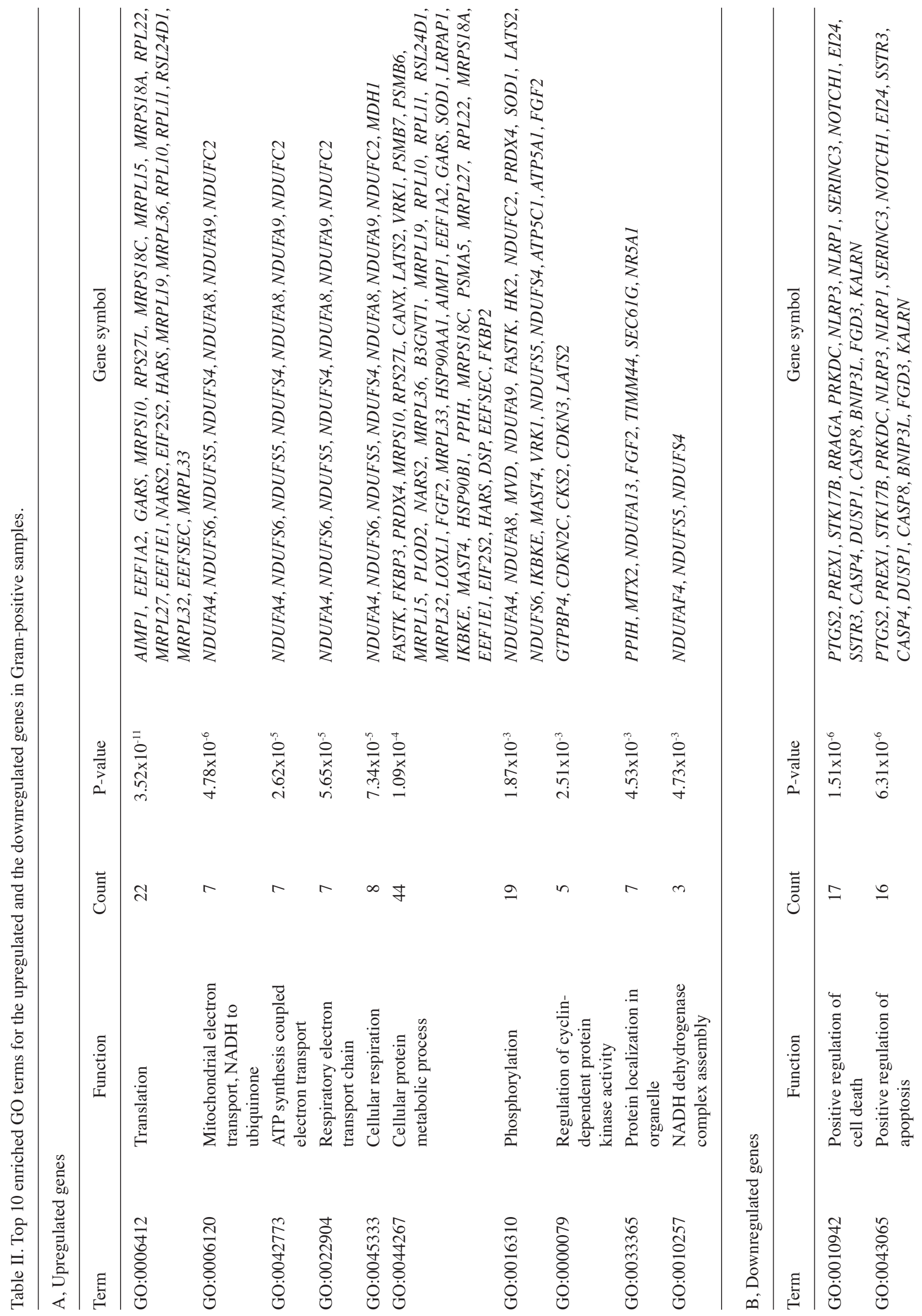




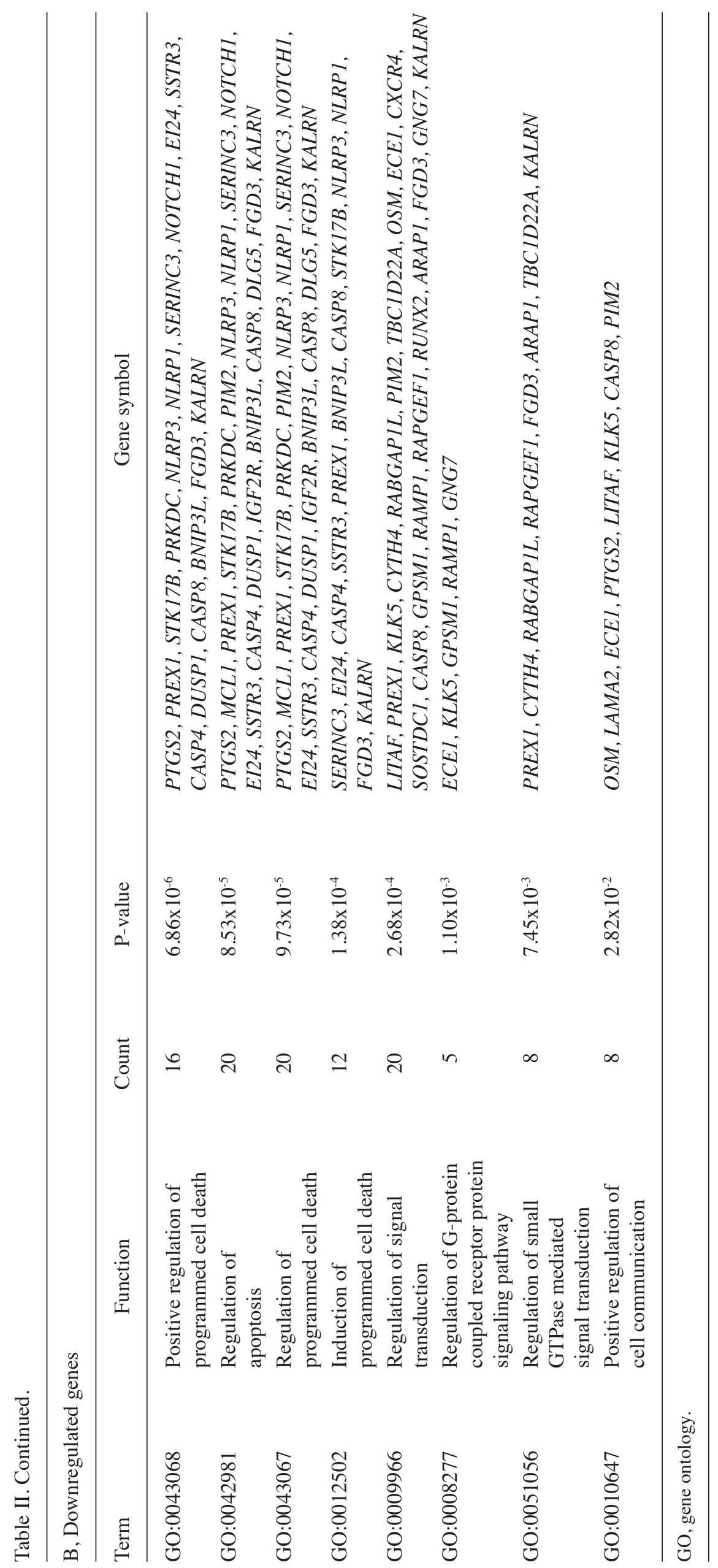




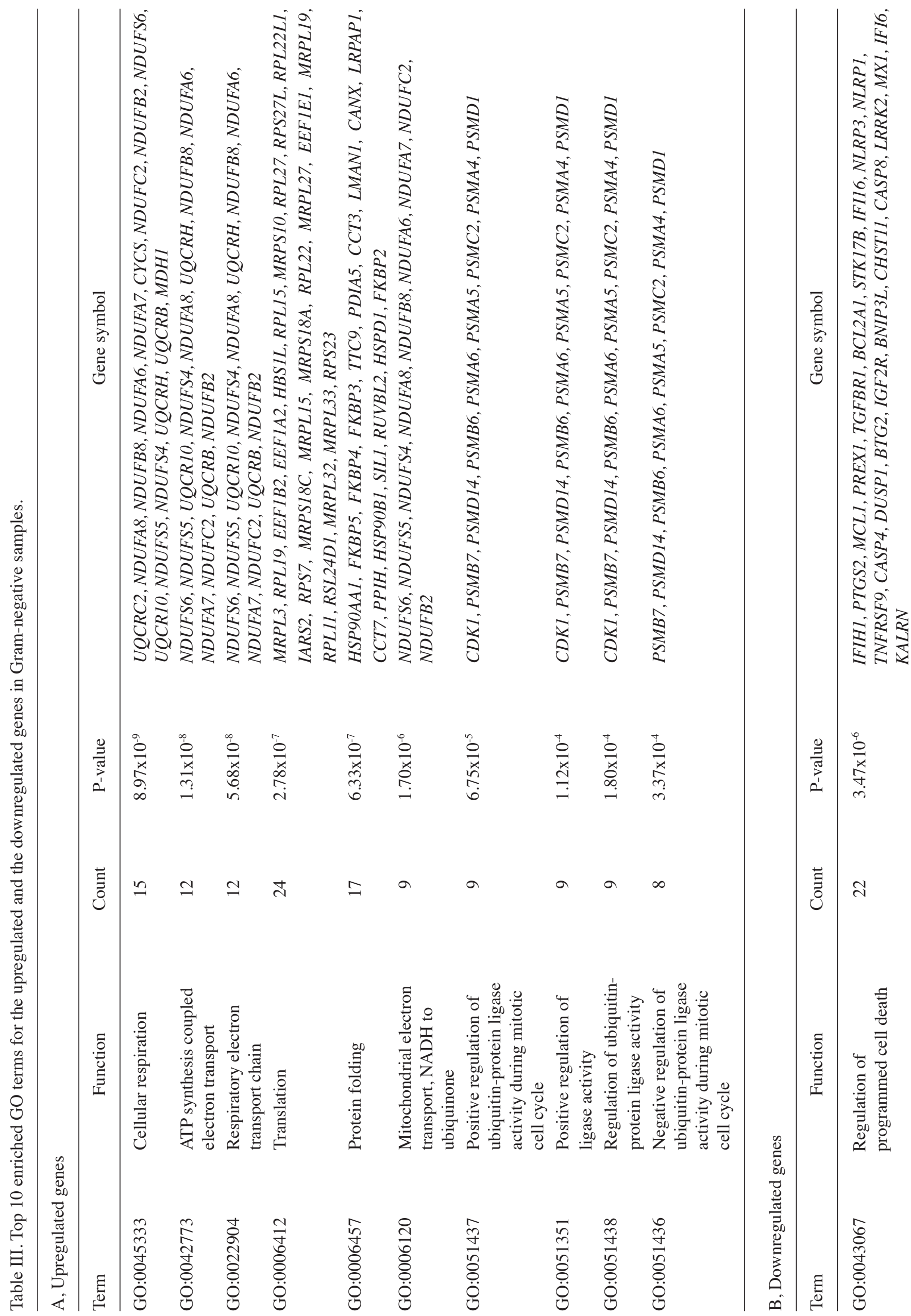




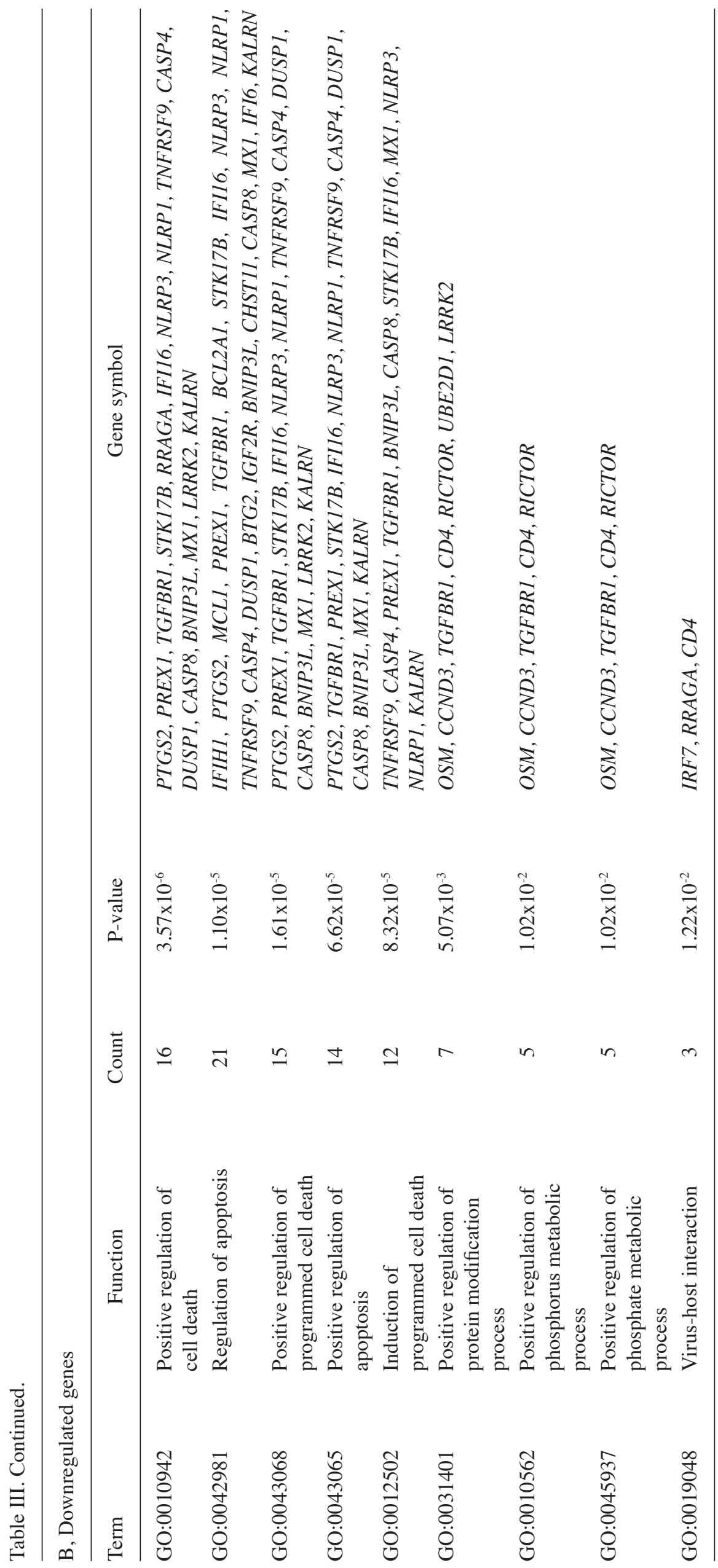




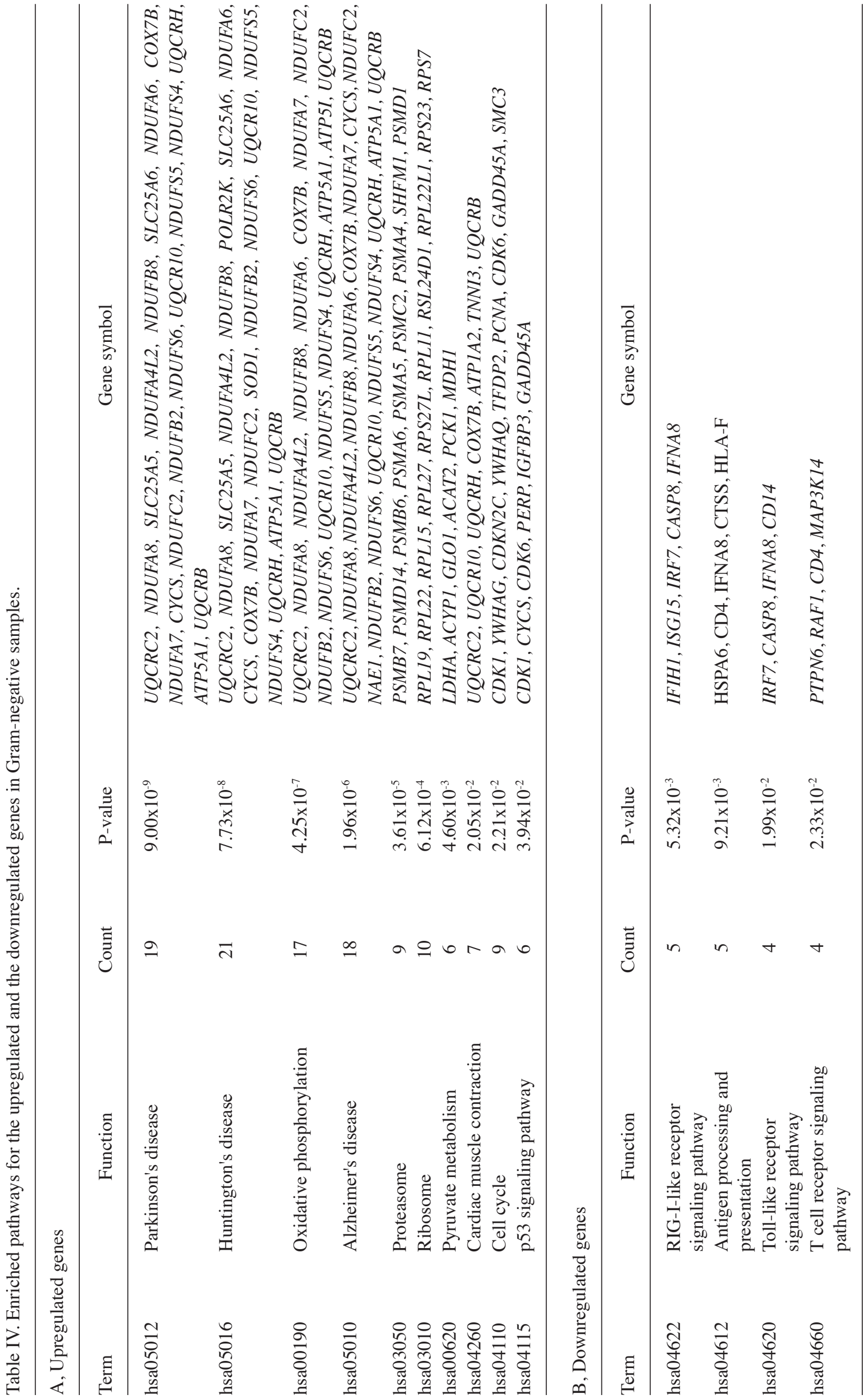




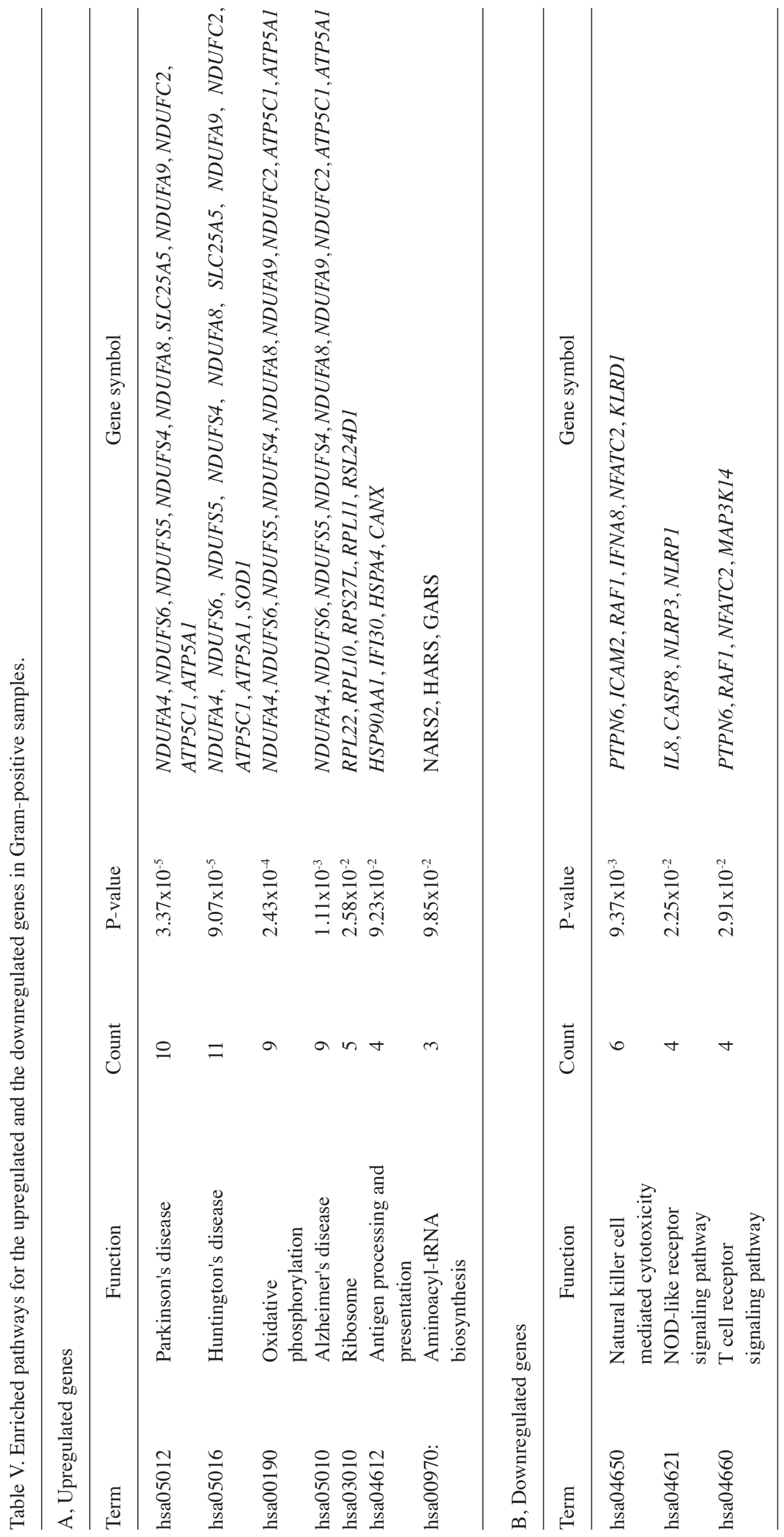




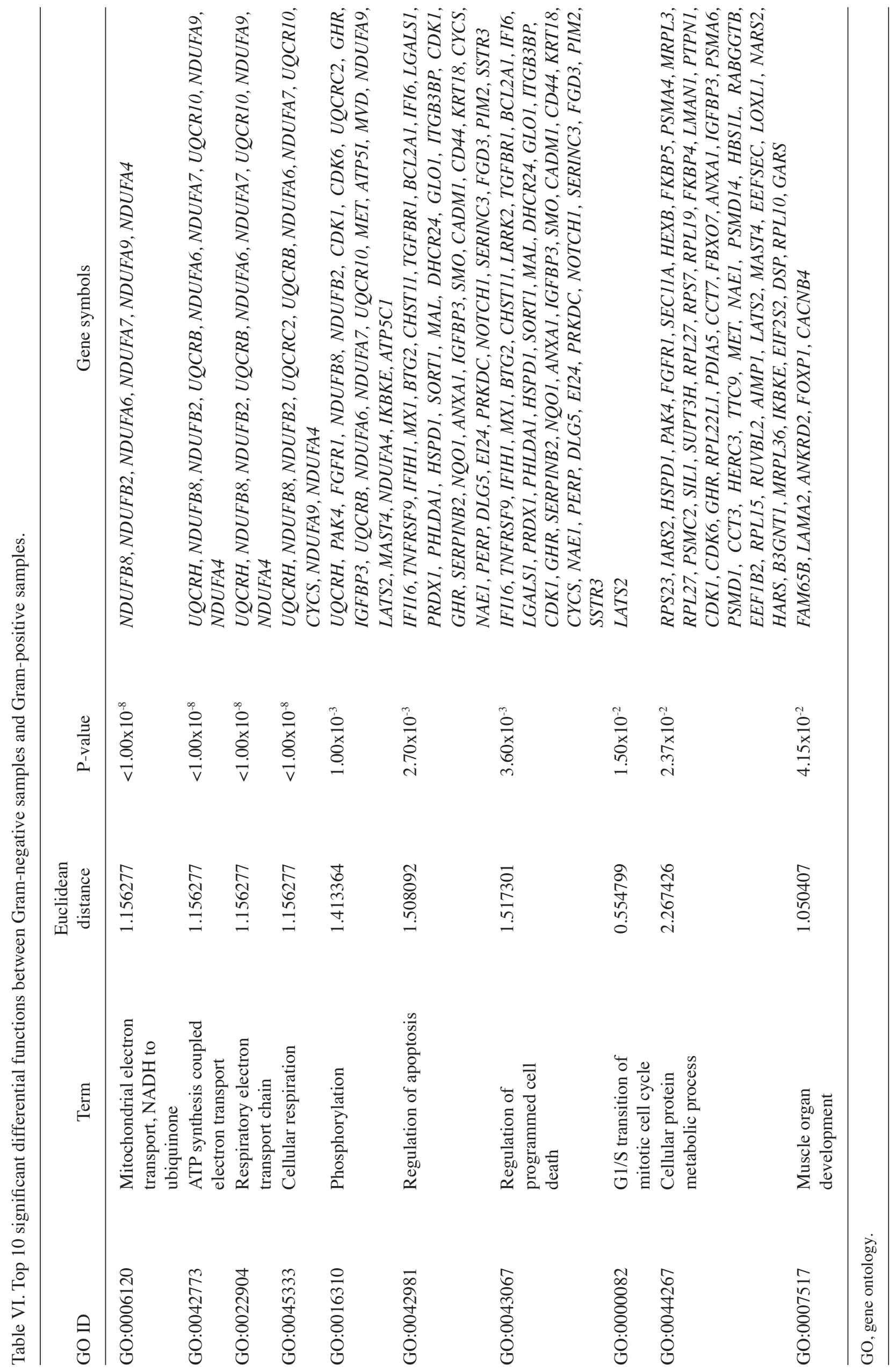




\section{References}

1. Taylor FB Jr, Kinasewitz GT and Lupu F: Pathophysiology, staging and therapy of severe sepsis in baboon models. J Cell Mol Med 16: 672-682, 2012

2. Brodská H, Malíčková K, Adámková V, Benáková H, Št’astná MM and Zima T: Significantly higher procalcitonin levels could differentiate Gram-negative sepsis from Gram-positive and fungal sepsis. Clin Exp Med 13: 165-170, 2013.

3. Deutschman C and Tracey K: Sepsis: Current dogma and new perspectives. Immunity 40: 463-475, 2014.

4. Lyle NH, Pena OM, Boyd JH and Hancock REW: Barriers to the effective treatment of sepsis: Antimicrobial agents, sepsis definitions, and host-directed therapies. Ann N Y Acad Sci 1323 101-114, 2014.

5. Hotchkiss RS and Karl IE: The pathophysiology and treatment of sepsis. N Engl J Med 348: 138-150, 2003.

6. Koh EM, Lee SG, Kim CK, Kim M, Yong D, Lee K, Kim JM, Kim DS and Chong Y: Microorganisms isolated from blood cultures and their antimicrobial susceptibility patterns at a university hospital during 1994-2003. Korean J Lab Med 27 265-275, 2007 (In Korean).

7. Glik J, Kawecki M, Gaździk T and Nowak M: The impact of the types of microorganisms isolated from blood and wounds on the results of treatment in burn patients with sepsis. Pol Przegl Chir 84: 6-16, 2012.

8. Tang BM, McLean AS, Dawes IW, Huang SJ, Cowley MJ and Lin RC: Gene-expression profiling of gram-positive and gram-negative sepsis in critically ill patients. Crit Care Med 36: 1125-1128, 2008.

9. Mahabeleshwar GH, Qureshi MA, Takami Y, Sharma N, Lingrel JB and Jain MK: A myeloid hypoxia-inducible factor $1 \alpha$-Krüppel-like factor 2 pathway regulates gram-positive endotoxin-mediated sepsis. J Biol Chem 287: 1448-1457, 2012.

10. Giamarellos-Bourboulis EJ, van de Veerdonk FL, Mouktaroudi M, Raftogiannis M, Antonopoulou A, Joosten LA, Pickkers P, Savva A, Georgitsi M, van der Meer JW and Netea MG: Inhibition of caspase-1 activation in Gram-negative sepsis and experimental endotoxemia. Crit Care 15: R27, 2011.

11. Kager LM, Weehuizen TA, Wiersinga WJ, Roelofs JJ, Meijers JC, Dondorp AM, van 't Veer C and van der Poll T: Endogenous $\alpha 2$-antiplasmin is protective during severe gram-negative sepsis (melioidosis). Am J Respir Crit Care Med 188: 967-975, 2013.

12. Kager LM, Wiersinga WJ, Roelofs JJ, de Boer OJ, Weiler H, van 't Veer C and van der Poll T: A thrombomodulin mutation that impairs active protein $\mathrm{C}$ generation is detrimental in severe pneumonia-derived gram-negative sepsis (melioidosis). PLoS Negl Trop Dis 8: e2819, 2014.

13. Afsari B, Geman D and Fertig EJ: Learning dysregulated pathways in cancers from differential variability analysis. Cancer Inform 13 (Suppl 5): S61-S67, 2014.

14. Calandra T and Cohen J; International Sepsis Forum Definition of Infection in the ICU Consensus Conference: The international sepsis forum consensus conference on definitions of infection in the intensive care unit. Crit Care Med 33: 1538-1548, 2005.

15. Murie C, Barette C, Lafanechère L and Nadon R: Control-plate regression (CPR) normalization for high-throughput screens with many active features. J Biomol Screen 19: 661-671, 2014.

16. Ritchie ME, Phipson B, Wu D, Hu Y, Law CW, Shi W and Smyth GK: limma powers differential expression analyses for RNA-sequencing and microarray studies. Nucleic Acids Res 43: e47, 2015 .

17. Chen H and Boutros PC: VennDiagram: A package for the generation of highly-customizable Venn and Euler diagrams in R. BMC Bioinformatics 12: 35, 2011.

18. Gene Ontology Consortium, Blake JA, Dolan M, Drabkin H, Hill DP, Li N, Sitnikov D, Bridges S, Burgess S, Buza T, et al: Gene ontology annotations and resources. Nucleic Acids Res 41 (Database issue): D530-D535, 2013.

19. Huang da W, Sherman BT and Lempicki RA: Systematic and integrative analysis of large gene lists using DAVID bioinformatics resources. Nat Protoc 4: 44-57, 2008.

20. Zhou J, Dong X, Zhou Q, Wang H, Qian Y, Tian W, Ma D and Li X: microRNA expression profiling of heart tissue during fetal development. Int J Mol Med 33: 1250-1260, 2014.
21. Zhai Y, Tchieu J and Saier MH Jr: A web-based tree view (TV) program for the visualization of phylogenetic trees. J Mol Microbiol Biotechnol 4: 69-70, 2002.

22. Weaver B and Wuensch KL: SPSS and SAS programs for comparing Pearson correlations and OLS regression coefficients. Behav Res Methods 45: 880-895, 2013.

23. Demchak B, Hull T, Reich M, Liefeld T, Smoot M, Ideker T and Mesirov JP: Cytoscape: The network visualization tool for GenomeSpace workflows. F1000Res 3: 151, 2014.

24. Kotera M, Moriya Y, Tokimatsu T, Kanehisa M and Goto S: KEGG and GenomeNet, New Developments, Metagenomic Analysis, pp329-339, 2015.

25. Liberti L, Lavor C, Maculan N and Mucherino A: Euclidean distance geometry and applications. Quantitative Biol 56: 3-69, 2012.

26. Bódai T, Altmann EG and Endler A: Stochastic perturbations in open chaotic systems: Random versus noisy maps. Phys Rev E Stat Nonlin Soft Matter Phys 87: 042902, 2013.

27. Schmidt MV, Paulus P, Kuhn AM, Weigert A, Morbitzer V, Zacharowski K, Kempf VA, Brüne B and von Knethen A: Peroxisome proliferator-activated receptor $\gamma$-induced T cell apoptosis reduces survival during polymicrobial sepsis. Am J Respir Crit Care Med 184: 64-74, 2011.

28. Chang K, Svabek C, Vazquez-Guillamet C, Sato B, Rasche D, Wilson S, Robbins P, Ulbrandt N, Suzich J, Green J, et al: Targeting the programmed cell death 1: Programmed cell death ligand 1 pathway reverses $\mathrm{T}$ cell exhaustion in patients with sepsis. Crit Care 18: R3, 2014.

29. Härter L, Mica L, Stocker R, Trentz O and Keel M: Mcl-1 correlates with reduced apoptosis in neutrophils from patients with sepsis. J Am Coll Surg 197: 964-973, 2003.

30. Galley H: Oxidative stress and mitochondrial dysfunction in sepsis. Br J Anaesth 107: 57-64, 2011.

31. Lee I and Hüttemann M: Energy crisis: The role of oxidative phosphorylation in acute inflammation and sepsis. Biochim Biophys Acta 1842: 1579-1586, 2014.

32. Petruzzella V, Vergari R, Puzziferri I, Boffoli D, Lamantea E, Zeviani $\mathrm{M}$ and Papa S: A nonsense mutation in the NDUFS4 gene encoding the $18 \mathrm{kDa}(\mathrm{AQDQ})$ subunit of complex I abolishes assembly and activity of the complex in a patient with Leigh-like syndrome. Hum Mol Genet 10: 529-535, 2001.

33. Ugalde C, Hinttala R, Timal S, Smeets R, Rodenburg RJ, Uusimaa J, van Heuvel LP, Nijtmans LG, Majamaa K and Smeitink JA: Mutated ND2 impairs mitochondrial complex I assembly and leads to Leigh syndrome. Mol Genet Metab 90: 10-14, 2007.

34. Bottley A, Phillips NM, Webb TE, Willis AE and Spriggs KA: eIF4A inhibition allows translational regulation of mRNAs encoding proteins involved in Alzheimer's disease. PLoS One 5: pii: e13030, 2010.

35. Dröse S and Brandt U: Molecular mechanisms of superoxide production by the mitochondrial respiratory chain. In: Mitochondrial Oxidative Phosphorylation. Springer, pp145-169, 2012.

36. Van Raamsdonk JM and Hekimi S: Superoxide dismutase is dispensable for normal animal lifespan. Proc Natl Acad Sci USA 109: 5785-5790, 2012.

37. Schulte J, Struck J, Köhrle J and Müller B: Circulating levels of peroxiredoxin 4 as a novel biomarker of oxidative stress in patients with sepsis. Shock 35: 460-465, 2011.

38. Owens KM, Kulawiec M, Desouki MM, Vanniarajan A and Singh KK: Impaired OXPHOS complex III in breast cancer. PLoS One 6: e23846, 2011.

39. Modena P, Testi MA, Facchinetti F, Mezzanzanica D, Radice MT, Pilotti S and Sozzi G: UQCRH gene encoding mitochondrial Hinge protein is interrupted by a translocation in a soft-tissue sarcoma and epigenetically inactivated in some cancer cell lines. Oncogene 22: 4586-4593, 2003.

40. Li Y, Pei J, Xia H, Ke H, Wang H and Tao W: Lats2, a putative tumor suppressor, inhibits G1/S transition. Oncogene 22: 4398-4405, 2003.

41. Yang QH, Liu DW, Long Y, Liu HZ, Chai WZ and Wang XT: Acute renal failure during sepsis: Potential role of cell cycle regulation. J Infect 58: 459-464, 2009. 